\title{
Die neuen Herausforderungen der Epidemiologie im politischen und sozialen Umfeld
}

\author{
Vom öffentlichen Gesundheitswesen bis zur Präventionspolitik
}

\author{
Clelia Di Serio
}

Psychotherapie-Wissenschaft 8 (1) 55-64 2018

www.psychotherapie-wissenschaft.info

CC BY-NC-ND

DOI: $10.30820 / 8242.11$

\begin{abstract}
Zusammenfassung: Ziel dieses Beitrags ist, einen breiten Überblick darüber zu geben, wie die Evolution biomedizinischer Informationen - aus epidemiologischer und aus gesundheitspolitischer Sicht - zwangsläufig zu einer Veränderung der Analysewerkzeuge und -methoden geführt hat.

Um den Herausforderungen zu begegnen, die mit der wechselnden Bevölkerungsdynamik, den neuen grossen Migrationsströmen, der Veränderung der Alterungsdynamik und der sehr schnellen Entwicklung der Medizin in Richtung «Precision Medicine» zusammenhängen, ist es notwendig, dass die gesellschaftlich-organisatorische Antwort des Sozialund Gesundheitssystems den Veränderungen des Fortschritts und den neuen Bedürfnissen angepasst wird.

Im Beitrag werden wir darlegen, wie mit dem Übergang von einer individuellen zu einer Perspektive von epidemiologisch typischer Population auch viele Paradigmen ändern, die mit einer Eigenschaft des Anhaltspunkts zusammenhängen. Wir positionieren uns in einem Modell-Typ «Global System», in dem die Integration zwischen verschiedenen Informationsquellen und Präventionsmassnahmen unerlässlich wird.
\end{abstract}

Schlüsselwörter: Öffentliche Gesundheit, Überwachung, Altern, Massendaten, psychische Gesundheit

\section{Einführung}

Es gibt zahlreiche Möglichkeiten, die grundlegenden Konzepte der Epidemiologie und ihre Rolle in der Gesellschaft zu erläutern. Seit den ersten Versuchen der Pockenimpfung, die der alten indischen Medizin zugeschrieben wurden, sind Epidemiologie, Volksgesundheit und Politik stets miteinander verbunden gewesen. Dies gilt insbesondere für Konfuzius, der bald herausgegeben hatte, was wir heute als «Leitlinien» für die Zubereitung von Lebensmitteln bezeichnen würden, um Krankheiten fernzuhalten, die «durch den Mund in uns gelangen». Hippokrates selbst beschrieb die abführende Wirkung von Vollkorngerste im Vergleich zu kleiefreier Gerste genau und die medizinische Fakultät von Salerno identifizierte in einigen Essgewohnheiten die Grundlage für die Pflege einer guten Gesundheit. Aber wenn wir Epidemiologie im engeren Sinne einführen wollen, müssen wir feststellen, dass diese keine «alte» Disziplin wie die anderen exakten Wissenschaften ist, sondern mit der Evolution der Gesellschaft verbunden ist.

Man kann von einem integrativen historischen Ansatz ausgehen, der die Geburt der Epidemiologie und der medizinischen Statistik parallel zur Entstehung und Entwicklung von Disziplinen wie Genetik und Informatik (Abbildung 1) ansetzt, die mit der Epidemiologie stark integriert sind. Oder wir können die Geburt der Epidemiologie als eng verknüpft mit den ersten relevanten Wahrscheinlichkeitstheorien des 17. Jahrhunderts von Pascal bis Fermat (1654) betrachten.
Aber der wahrscheinlich beste Weg, die Entstehung der Disziplin der Epidemiologie richtig zu datieren, besteht in ihrer Verknüpfung mit den ersten demographischen Datenerfassungen. Letztere führten zur Berechnung der ersten Sterbetafel durch einen der Begründer der modernen Demographie, John Graunt, der 1662 das Buch «Natural and political observations mentioned in a following index» verfasste, in dem er Geburtenrate, Sterblichkeit und Geschlechterdifferenzierung der Londoner Bevölkerung auswertete.

Die Arbeit wurde von De Moivre fortgesetzt, der 1.725 Begriffe wie Zensur und die ersten linearen Risikofunktionen einführte und entwickelte. Gompertz und Makeham vertieften diese später weiter und implementierten reale Modelle für die unmittelbare Todesgefahr.

Neben der Entwicklung statistischer Verfahren zur prädiktiven Analyse von Daten wurden auch erste Modelle der Verbreitung von Krankheiten entwickelt. Zu diesen zählt das Modell von John Snow, der als Vater der Epidemiologie anerkannt wird. 1854 gelang es ihm dank der räumlichen Analyse der Cholera-Anhäufungen in London, die Epidemie zu stoppen, indem er die für die Epidemie verantwortliche Ursache in einem der beiden Wasserversorgungen Londons (Broad Street Water Pump) identifizierte und ausser Betrieb setzte, ohne den Vibrio cholerae zu kennen.

Gleichgültig, mit welchem Ansatz man sich der Epidemiologie nähert, ob vom statistisch-mathematischen oder vom medizinisch-demographischen Standpunkt, 


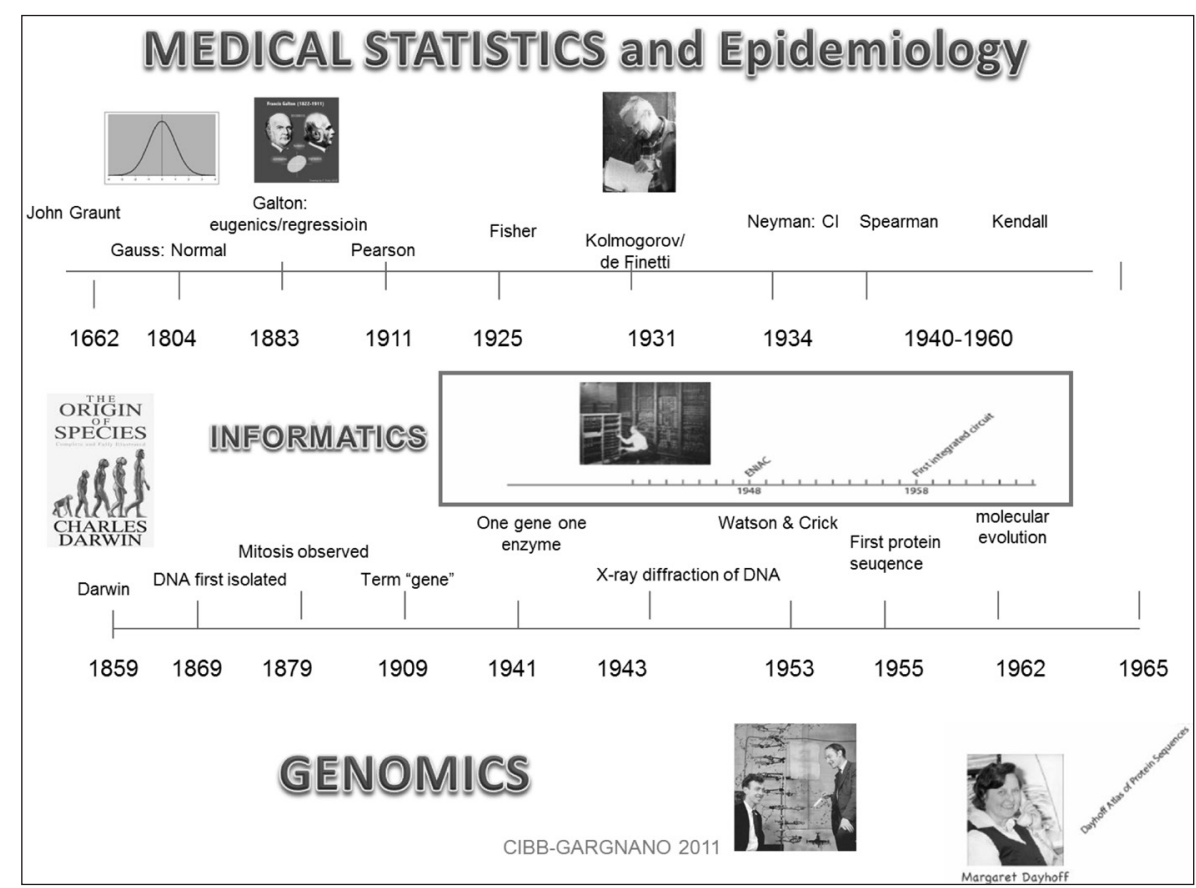

Abb. 1: Die Abbildung zeigt, wie sich die statistische Disziplin parallel und in integrierter Form mit Disziplinen wie Genomik und Informatik entwickelt hat.

Tatsache ist: Die Disziplin entwickelt sich erst ab Mitte des 16. Jahrhundert, als sich eine deutliche Zunahme des der durchschnittlichen Lebenserwartung des Menschen abzeichnete.

Erst von diesem historischen Moment an beginnt der Begriff der «Vorhersage», das heißt "voraus sehen» (prae-dicere, im Voraus behaupten) Sinn zu machen, an der Grenze zu esoterischen Disziplinen. Begriff, der sich dann in die eher mathematische Idee des «vorher Sehens» oder der «Voraussicht» (prae videre, voraussehen) mit den prädiktiven linearen Modellen von Francis Galton und der Regressionstheorie wandelt. Heutzutage wird alles, was epidemiologische Studien in biomedizinischen Disziplinen betrifft, von der klinischen bis zur Grundlagenforschung, Grundlage für «Prävention» (prae-venire, «vorher kommen»), das heißt für Tätigkeiten und Massnahmen, die auf die «Früherkennung» von Krankheiten abzielen; dadurch wird die Epidemiologie unmittelbar in die Gesellschaft und den öffentlichen Gesundheitsdienst integriert.

Tatsächlich sind der Versuch, das Auftreten einer Krankheit, eines bestimmten Symptoms oder deren Verschlimmerung bzw. Rückfalls bis hin zu einem möglichen tödlichen Ereignis zu verhindern sowie die zu diesem Zweck eingesetzten Verfahren grundlegende Aspekte der Politik der Gesundheitsintervention.

\section{Epidemiologie: individuelle oder bevölkerungsbezogene Perspektive?}

Im Bereich der öffentlichen Gesundheit lassen sich zwei Standpunkte unterscheiden, die nicht immer übereinstimmen und deren Konfrontation zu ethischen und po- litischen Konflikten führen kann: eine individuelle Perspektive und eine Bevölkerungsperspektive. Die individuelle Perspektive fokussiert sich auf die Gesundheits-, Risikound Expositionsfaktoren des Probanden und das Vorhandensein möglicher Abhängigkeitsmechanismen zwischen diesen Faktoren, die Krankheiten im Individuum verursachen können. Koronare Herzkrankheit, Krebs, Herzinfarkt, Bluthochdruck, Asthma, neurodegenerative Erkrankungen, all dies sind potenziell katastrophale Ereignisse im Leben des Einzelnen.

Nach moderner Auffassung beschäftigt sich die medizinische Statistik damit, der modernen Medizin die Beweisgrundlagen zu liefern: Anfänglich mit der «translationalen Medizin» (Translational Medicine), dann mit der so genannten "personalisierten Medizin» (Personalized Medicine). Heute spricht man hingegen von «Präzisionsmedizin» (Precision Medicine).

Die Entwicklung der «Datentechnologie» hat auch zur Entstehung neuer Interaktionsprozesse zwischen Epidemiologie, Überwachung, Prävention und öffentlicher Gesundheit geführt, wie in Abbildung 2 dargestellt wird.

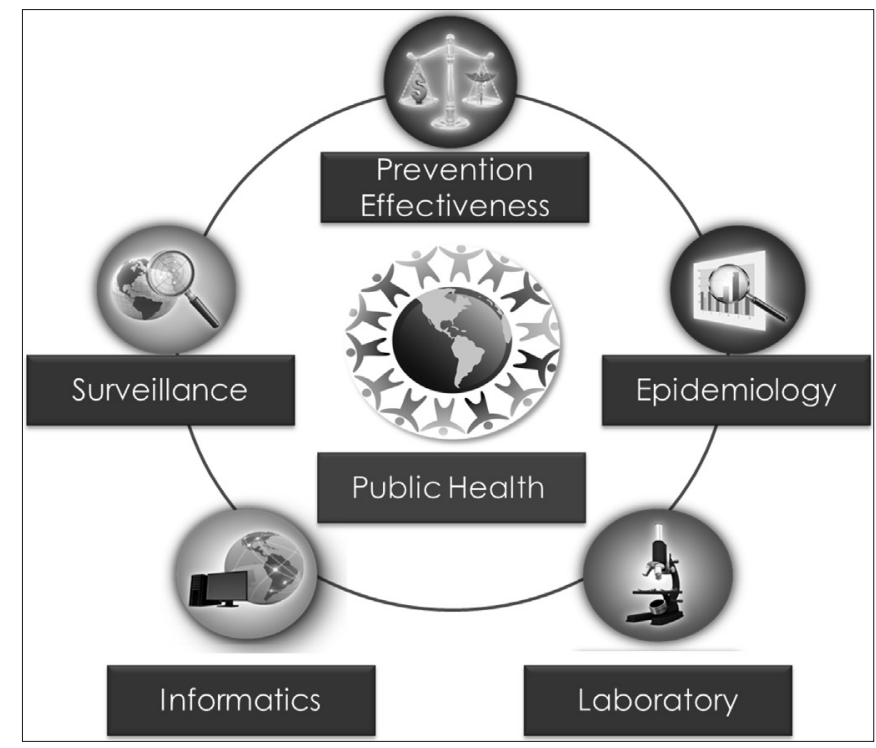

Abb. 2: Interaktionsprozess zwischen den Disziplinen im Gesundheitswesen.

Was steht hinter diesem ständigen Perspektivenwechsel der Medizin und wie hat die medizinische Statistik ihre Paradigmen entsprechend verändert? Der wesentliche Punkt ist die Veränderung der Art der Daten, die die Welt der Biomedizin zu einem einzigen «globalen System» (Global System) gemacht hat. 
Bis vor wenigen Jahrzehnten war das Problem der Verwendung von Statistiken in der Medizin ein Problem des «Nachweis Erbringens» durch einen reinen «Induktionsprozess»: In einem Inferenzprozess wurde, ausgehend von einer relativ kleinen Stichprobe, mithilfe statistischer Inferenzinstrumente eine «Inferenz» der Bevölkerung erstellt, um dann von «evidenzbasierter Medizin» (Evidence Based Medicine EBM) zu sprechen. Heute, in der Ära von BIG DATA, auf die wir im letzten Abschnitt zurückkommen werden, hat sich das Statistik-Paradigma in der Medizin verändert und die Medizin ist zu einem integrierten Informationssystem geworden. Mit dem Aufkommen von Genomik, Proteomik, mit den grossen Konsortien und dem Föderalismus biomedizinischer Daten eröffnet sich eine enger gefasste «deduktive» Perspektive der Disziplin.

In der Tat geht es in der "translationalen Medizin» darum, heterogene Datenquellen und interdisziplinäres Wissen, das in der Grundlagen- und klinischen Forschung nutzbar ist, zusammenzuführen und transversal zu integrieren. Ziel ist, die Kenntnis der Pathologien zu verbessern, um einen «Deduktionsprozess» zu initiieren, bei dem man von der allgemeinen Pflege zu einer «personalisierten» und auf den Patienten abgestimmte Therapie zurückzukehren versucht. In der modernen medizinischen Auffassung spricht man eher von «Präzisionsmedizin», um eine im Vergleich zur personalisierten Medizin grössere Dimension wiederzufinden. Zumal selbst massgebliche Stellen, wie der National Research Council (Nationaler Forschungsrat) festgestellt haben, dass hinsichtlich der Nachhaltigkeit des Gesundheitssystems eine nur anhand der Merkmale eines Individuums patentierte Therapie unwirtschaftlich und zuweilen auch moralisch fragwürdig ist.

Heute spricht man lieber von "Präzisionsmedizin» oder «Präzision», bei der Therapien auf der Basis von

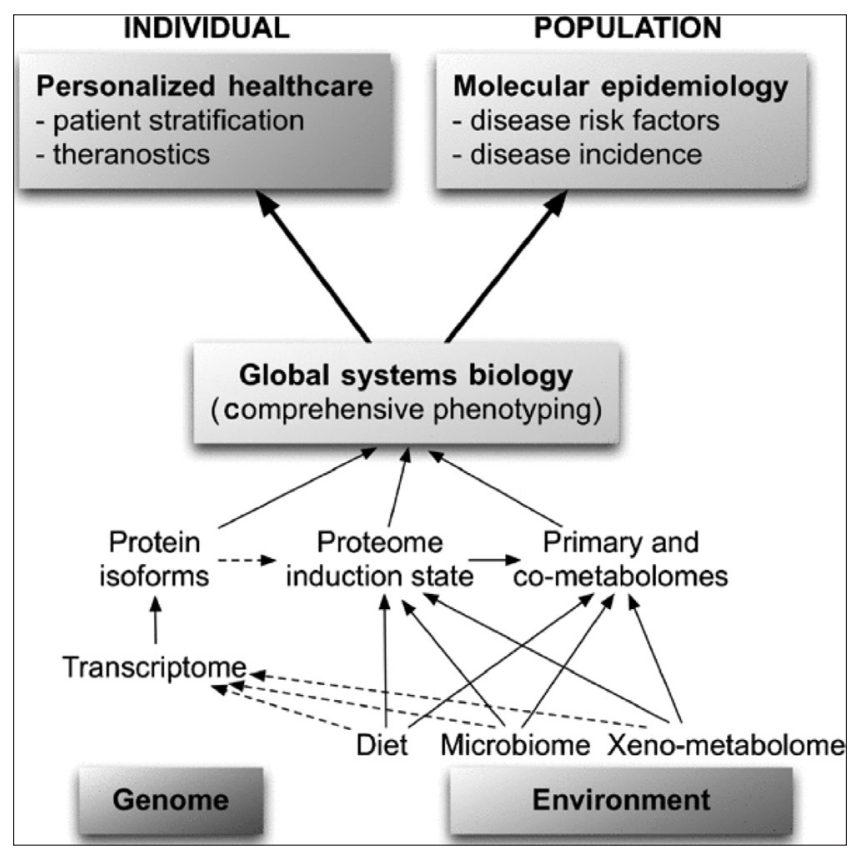

Abb. 3: Beziehungen zwischen Systembiologie, personalisierter Medizin und molekularer Epidemiologie.

genetischen, ökologischen und Lifestyle-Faktoren patentiert werden; und zwar, um zu Therapien einzusetzen, die sich an eine "Gruppe» von Patienten anpassen lassen, statt an das einzelne Individuum. Es lässt sich dennoch nachvollziehen, wie die «individuelle» Perspektive zu Massnahmen im Bereich der öffentlichen Gesundheit führen könnte, die stark von denjenigen abweichen, die durch eine «Bevölkerungsperspektive» identifiziert wurden, wie in Abbildung 3 dargestellt wird.

Die authentische Bedeutung der Epidemiologie, die sie

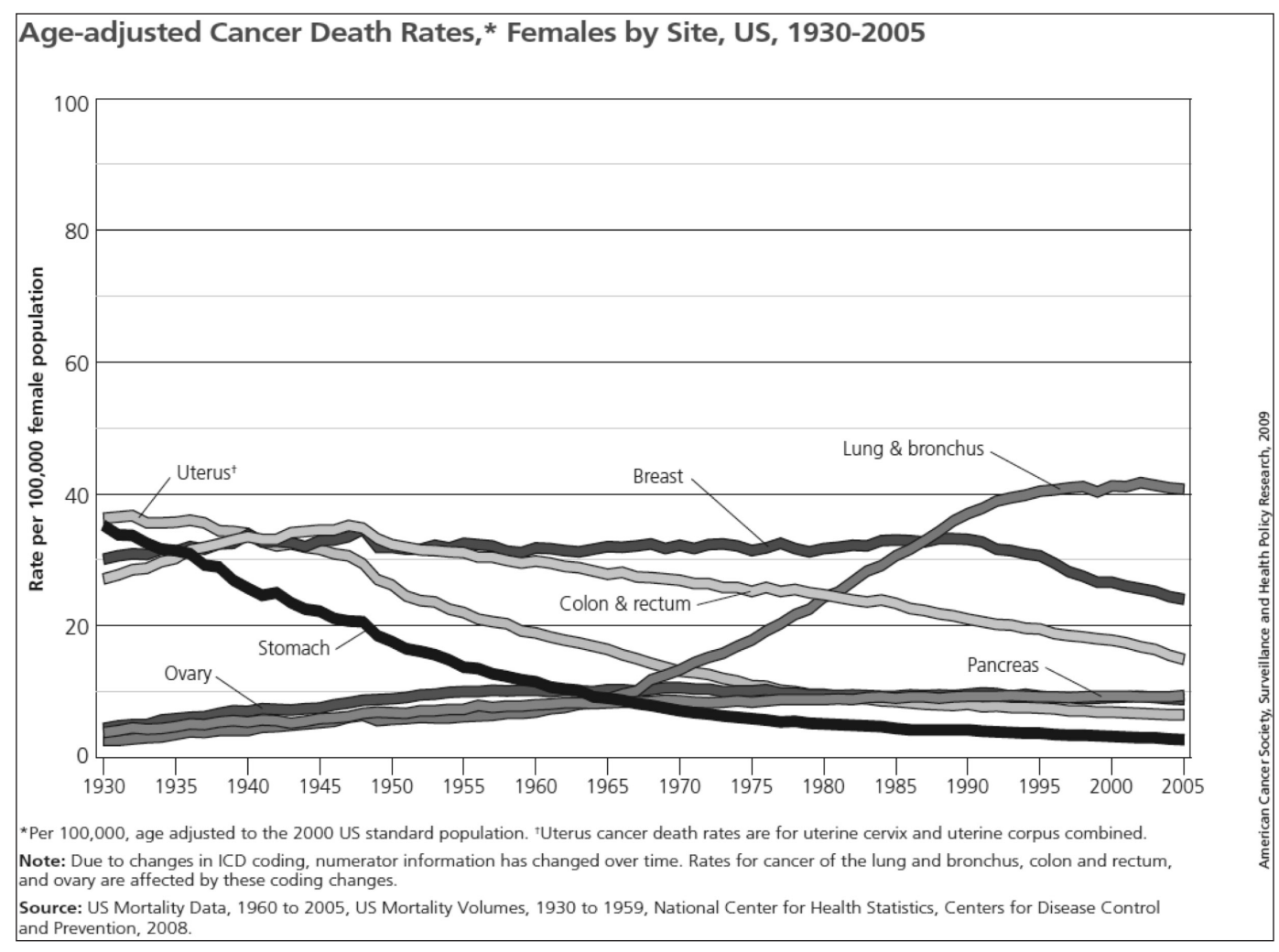

Abb. 4: Die Veröffentlichung der American Cancer Society «Cancer Facts \& Figures» basiert auf Daten des National Center for Health Statistics und des Bureau of the Census. 
auch von der medizinischen Statistik unterscheidet, liegt in ihrer Etymologie, das heißt im griechischen «epi-demologos», Studium der Bevölkerung. Die Bevölkerungsperspektive fokussiert sich auf eine Pathologie hinsichtlich einer grossen Anzahl von Individuen, bis hin zu den sogenannten Massenepidemien. Die Exposition gegenüber Risikofaktoren wird im Rahmen der Exposition von Menschengruppen bewertet.

Abbildung 4 zeigt die Sterblichkeitsraten von sieben Krebsarten [Kolon und Rektum, Leukämie, Leber, Lunge und Bronchien, Bauchspeicheldrüse, Prostata und Magen] bei Männern in den USA in einem Zeitraum von 1930 bis 2005. Was lesen wir in einer solchen Grafik, die kennzeichnend ist für Epidemiologie? Einerseits sehen wir der Entwicklung von sieben Tumorarten über die Zeit, zum Beispiel in den Longitudinaldiagrammen, wo wir gegenüber der Stabilität fast aller Tumore den dramatischen Anstieg des Lungenkrebses feststellen und insbesondere einen Wendepunkt identifizieren können.

Wir sehen, dass sich der Spitzenwert von 1965 aus epidemiologischer Sicht als fundamentale Interpretation für die Forschung nach den Ursachen des Anstiegs erweist. In jenen Jahren wurde die Tabak-Eskalation von der WHO als «die tragischste Entwicklung in der modernen Geschichte der Medizin» (Jan Stjernsward, WHO) erkannt. Seither wurde auf globaler gesellschaftlicher Ebene eine Kampagne gegen das Rauchen gestartet, die bis heute andauert. Sie begann mit dem Verbot der expliziten Werbung, dem Hinzufügen von abschreckenden Sätzen auf den Zigarettenpackungen um schliesslich das obligatorische Rauchverbot an jedem öffentlichen Ort einzuführen.

Der Lungenkrebs stellt einen klaren Beweis dar für die Notwendigkeit einer Bevölkerungsperspektive zur Eindämmung einer als «Massenepidemie». Die Bevölkerungsperspektive untersucht daher nicht so sehr die klinisch-biologischen Risikofaktoren für das Individuum, sondern bezieht sich vielmehr auf die Umweltfaktoren und die damit verbundenen Veränderungen im politischen Gesundheitssystem.

Ein weiteres Beispiel von Pandemie im 20. Jahrhundert ist die HIV/AIDS (Humaner Immundefizienz-Virus bzw. erworbenes Immunschwächesyndrom). In der «individuellen Perspektive» auf HIV konzentrieren sich die Behörden auf das Vorbeugen und Verhindern von riskanten Verhaltensweisen des Einzelnen. Tatsächlich haben die "Centers for Disease Control and Prevention (CDC)» etliche Millionen

Abb. 5: Nach Geschlecht geschichtetes Bevölkerungswachstum.

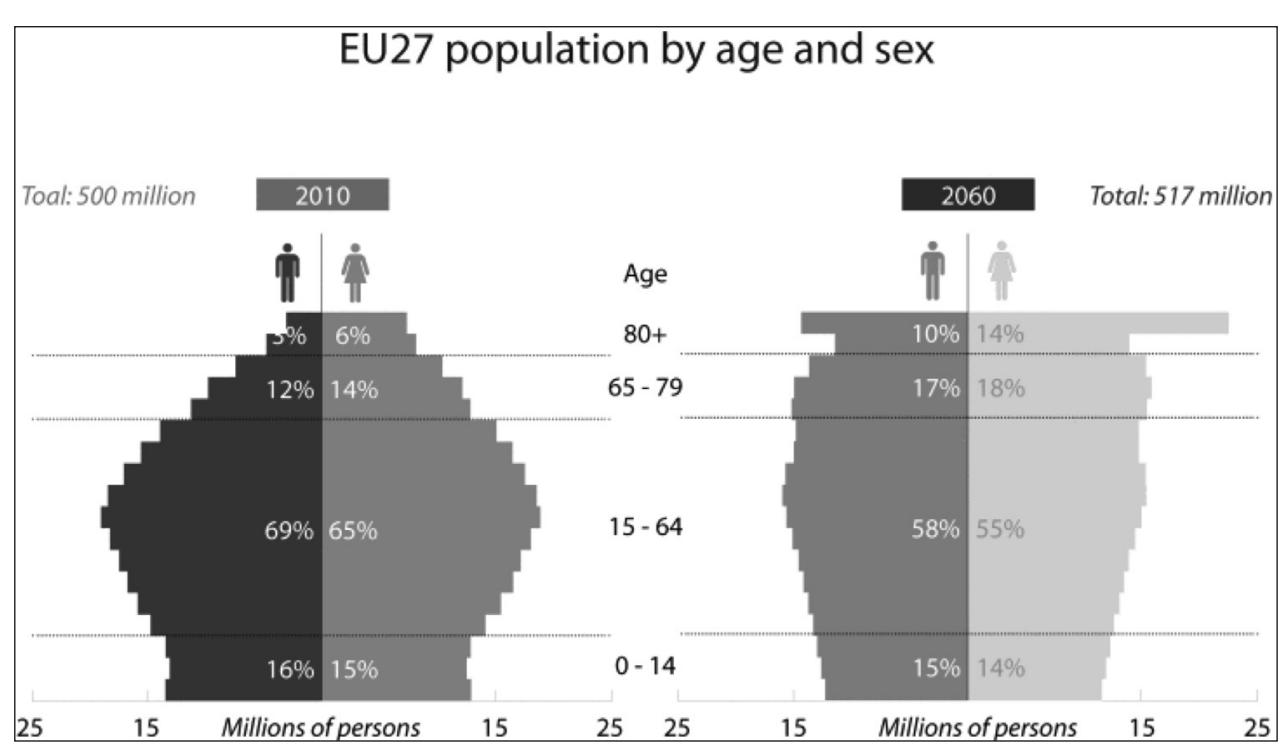

Dollar in erzieherische Programme investiert, einschliesslich des Erwerbs von HIV-Tests, die auf breiter Ebene an die Bevölkerung verteilt werden. Eine Bevölkerungsperspektive hingegen basiert auf sozioökonomischen und demographischen Faktoren, die zur Verbreitung des Virus beigetragen haben (1). Dazu zählen die Einwanderungsströme von Arbeitnehmern aus Gebieten mit hoher Pandemierate - wie dem südöstlichen Teil von Subsahara-Afrika (2), wo die untergeordneten afrikanischen Frauen in den Stämmen vom Kindesalter an zu promisken Verhaltensweisen gezwungen werden, die das Krankheitsrisiko stark erhöhen (3); die Explosion des Flugverkehrs und die Globalisierung im Allgemeinen haben zu einer raschen Ausbreitung des Virus beigetragen.

Die individuelle Perspektive beruht auf der «Diagnostik» und strebt nach einer "massgeschneiderten" Behandlung des Patienten. Die Bevölkerungsperspektive befasst sich mit «Screening», Massenprävention sowie mit der Ermittlung der primären und sekundären Interventionsstufe der Prävention.

\section{Altersepidemiologie und Überwachung}

Der Alterungsprozess tritt in bestimmten Entwicklungsphasen der Bevölkerung auf und ist das Ergebnis eines Rückgangs der Fruchtbarkeit bei gleichzeitiger Erhöhung von Lebensdauer und Lebenserwartung. Diese Veränderungen in der Zusammensetzung der Bevölkerung, obwohl absehbar, haben sich schneller vollzogen als erwartet. Sie lassen daher oft keine effiziente Anpassung des sozioökonomischen Systems an die Entwicklung zu. Folglich wurden sie von den meisten Institutionen als negative Phänomene eingestuft und lange als typisches «Problem des Alterns» betrachtet.

Seit den 1970er Jahren hat sich diese Sichtweise langsam geändert, vor allem dank dem Demographen Edward Rosset. Er führte 1978 eine Untersuchung durch, die eine höhere Entwicklung der Bevölkerungen, verbunden mit einem raschen Wachstum der durchschnittlichen Lebensdauer offenbarte. 
Der Eurostat-Bericht 2010-2060 zeigt eine sehr schnelle Wachstumsdynamik, wie aus Abbildung 5 hervorgeht; wobei ein besonderer Spitzenwert für ältere Frauen sichtbar ist, für die ein starkes Wachstum zu erwarten ist. Obgleich die Auswirkungen der sozioökonomischen Entwicklung weitgehend auf den Frauen lasten, sind die spezifischen Politiken immer noch wenig geschlechtsspezifisch differenziert. Sie passen sich nur langsam an das grössere Engagement der Frauen auf dem Arbeitsmarkt, ausserhalb der Familie, und an die Schwächung des Familien- und Elterngefüges an. Dies führt dazu, dass Frauen sich zuerst um ihre Kinder kümmern müssen und dann oft gleichzeitig den Ehemann und die Eltern.

In Übereinstimmung mit dem «Ageing and Life Course» der WHO hat sich gezeigt, wie eng das Altern mit Woblbefinden verbunden ist. Tatsächlich wirft die demografische Entwicklung viele wichtige Fragen im Zusammenhang mit der Nachhaltigkeit der Sozial- und Gesundheitssysteme auf, die mit einer steigenden Nachfrage nach Dienstleistungen und Pflegedienstleistungen konfrontiert sind, insbesondere mit langfristiger Pflege oder dauerhafter Pflege im Zusammenhang mit Behinderung älterer Menschen.

Die Prognosen der UNO (1) zeigen, dass im Jahr 2050 fast 2 Milliarden Menschen über 60 Jahre alt sein werden, also mehr als dreimal so viele wie im Jahr 2005; dies entspricht etwa einem Viertel $(22 \%)$ der Weltbevölkerung, wobei einige Länder in die entgegengesetzte Richtung tendieren, wie die Schweiz, wo der Prozentsatz auf 16 Prozent sinkt.

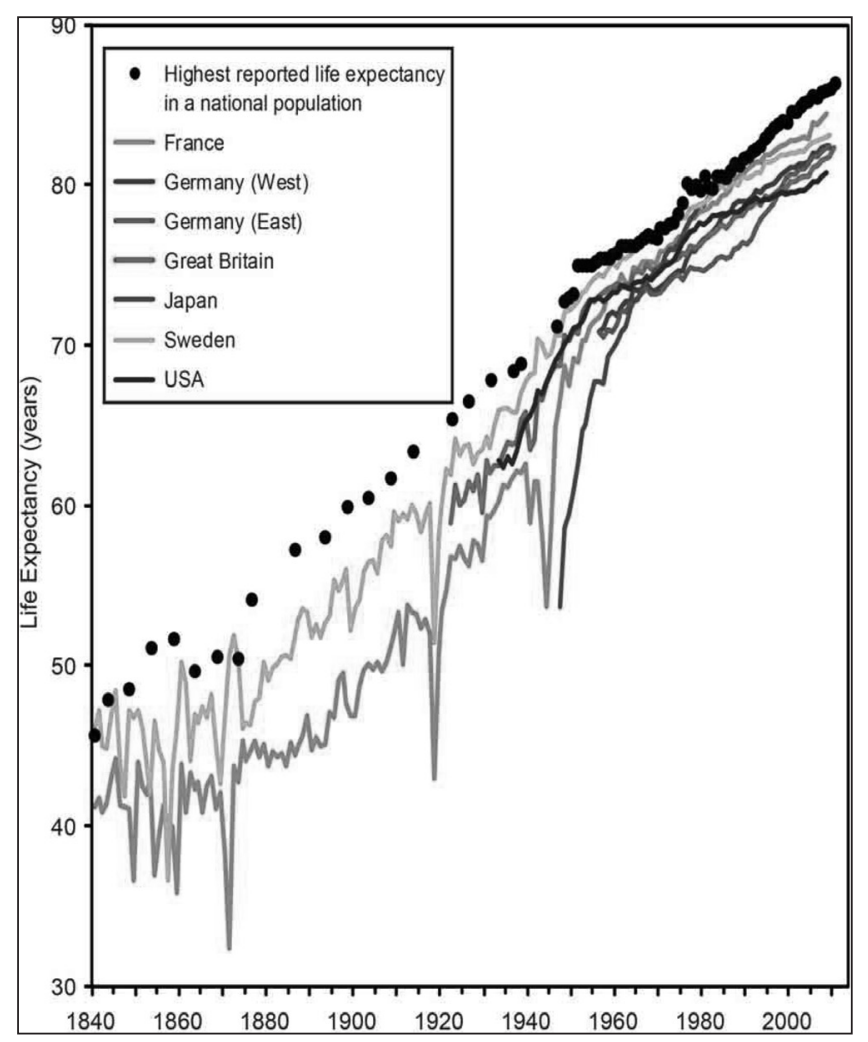

Abb. 6: Quelle: Highest reported life expectancy for the years 1840 to 2000 from online supplementary material to Oeppen J, Vaupel JW. Broken limits to life expectancy. Science 2002, S. 296.
Insbesondere in den 27 EG-Ländern wird ein dramatischer Anstieg der Bevölkerung über 64 Jahre erwartet, die sich in den nächsten 40 Jahren von fast 87 Millionen auf rund 148 Millionen Menschen praktisch verdoppeln wird (2). Abbildung 6 veranschaulicht diese Entwicklung in einigen der wichtigsten europäischen Länder (http:// www.who.int/ageing/en/).

Der Anstieg der durchschnittlichen Lebenserwartung, der bis zum Jahr 2050 auf sechs Jahre geschätzt wird, macht daher eine sehr gezielte sozialmedizinische Interventionspolitik erforderlich. Diese umfasst auch die Förderung einiger Berufsfiguren wie die Betreuer für ältere Menschen und die Stärkung der Gesundheitsfürsorge in Bezug auf die mit dem Altern verbundenen Pathologien.

Eurostat hat darauf hingewiesen, dass die Mehrheit der europäischen Staaten noch nicht auf eine Politik des aktiven Alterns vorbereitet sind. Dies ist eine Politik, bei der ältere Menschen, die bei guter Gesundheit sind, zu einem aktiven und nicht nur passiven Teil der Gesellschaft werden können. Die bedeutet, dass sie wo immer möglich an allen wirtschaftlichen, kulturellen und sozialen Gütern der Gesellschaft teilnehmen, die deshalb anders ausgerichtet sein müssen.

Der globale Charakter und die enormen Auswirkungen des Phänomens erfordern koordinierte Reaktionen der verschiedenen Staaten, um sektorübergreifende Politiken zu fördern, die ein breites Spektrum sozialer und wirtschaftlicher Fragen abdecken.

\section{Die Rolle der Überwachung in der Gesellschaft der psychischen Störungen}

Wenn man von «Überwachung» spricht, kommt man nicht umhin, die entscheidende Rolle der öffentlichen Gesundheitsversorgung bei der Förderung der "psychischen» Gesundheit auf der Grundlage von Überlegungen zur Lebensqualität, zur Nachhaltigkeit eines sozioökonomischen Systems und zur öffentlichen Gesundheit im Allgemeinen in Betracht zu ziehen. Probleme der psychischen Gesundheit sind zu einer der Hauptursachen für Fehlbleiben am Arbeitsplatz und Frühpensionierung in Europa geworden. Darüber hinaus führen der derzeitige wirtschaftliche Abschwung und seine Auswirkungen auf den Arbeitsmarkt zu Problemen bei der Beschäftigung und bei der Lebensqualität von Menschen mit psychischen Störungen und ihren Familien.

Die Veröffentlichung von Oms Europa «Mental health and well-being at the workplace - protection and inclusion in challenging times» (Psychische Gesundheit und Wohlbefinden am Arbeitsplatz - Schutz und Eingliederung in schwierigen Zeiten) ... Tatsächlich leiden weltweit etwa 450 Millionen Menschen an psychischen Erkrankungen, wenn man davon ausgeht, dass ein Viertel der Weltbevölkerung schätzungsweise irgendwann in ihrem Leben an einer psychischen Störung oder Verhaltensstörung leidet. Psychische Erkrankungen machen in den Vereinigten Staaten, Kanada und Westeuropa etwa 25 Prozent aller Behinderungen aus und sind, wenn man auch kognitive 
Erkrankungen mit einbezieht, eine der Hauptursachen für vorzeitigen Tod.

Zum Beispiel wird in den Vereinigten Staaten geschätzt, dass die Prävalenz im Leben von Erwachsenen von Angst- und Depressionsstörungen bei 29 Prozent, von Kontrollverlust und Zwangsstörungen bei 25 Prozent, von Stimmungsschwankungen bei 21 Prozent und von anderen Störungen bei 46 Prozent liegt. Die Auswirkungen psychischer Störungen sind während des gesamten Lebens auf allen sozioökonomischen Ebenen feststellbar.

Die Kosten für das Gesundheitssystem sind ebenfalls enorm. In den USA werden die geschätzten jährlichen Kosten auf 150 Milliarden Dollar pro Jahr ausschliesslich Forschungskosten geschätzt. Die nächste Abbildung zeigt den Zusammenhang zwischen Prävalenz und Behandlung bei einer der wichtigsten psychischen Störungen, der Depression. sionen und Schizophrenie eine 40-60 Prozent grössere Wahrscheinlichkeit als der Rest der Bevölkerung auf einen vorzeitigen Tod, und zwar aufgrund von körperlichen Gesundheitsproblemen, die oft nicht behandelt werden (wie Krebs, Herz-Kreislauf-Erkrankungen, Diabetes oder HIV-Infektion), und wegen Selbstmord. Letzterer ist die zweitwichtigste Todesursache bei jungen Menschen weltweit.

Zwölf Psychische Störungen wirken sich häufig auch andere Krankheiten aus wie Krebs, Herz-Kreislauf-Erkrankungen und HIV/AIDS-Infektionen und werden ihrerseits von diesen beeinflusst. Daher erfordern sie gemeine, übereinstimmende Pflege und eine aktive Mobilisierung der Ressourcen. So hat sich beispielsweise gezeigt, dass Depressionen zu einer Prädisposition für Herzinfarkt und Diabetes führen können, was wiederum das Risiko des Auftretens von Depressionen erhöht. Es

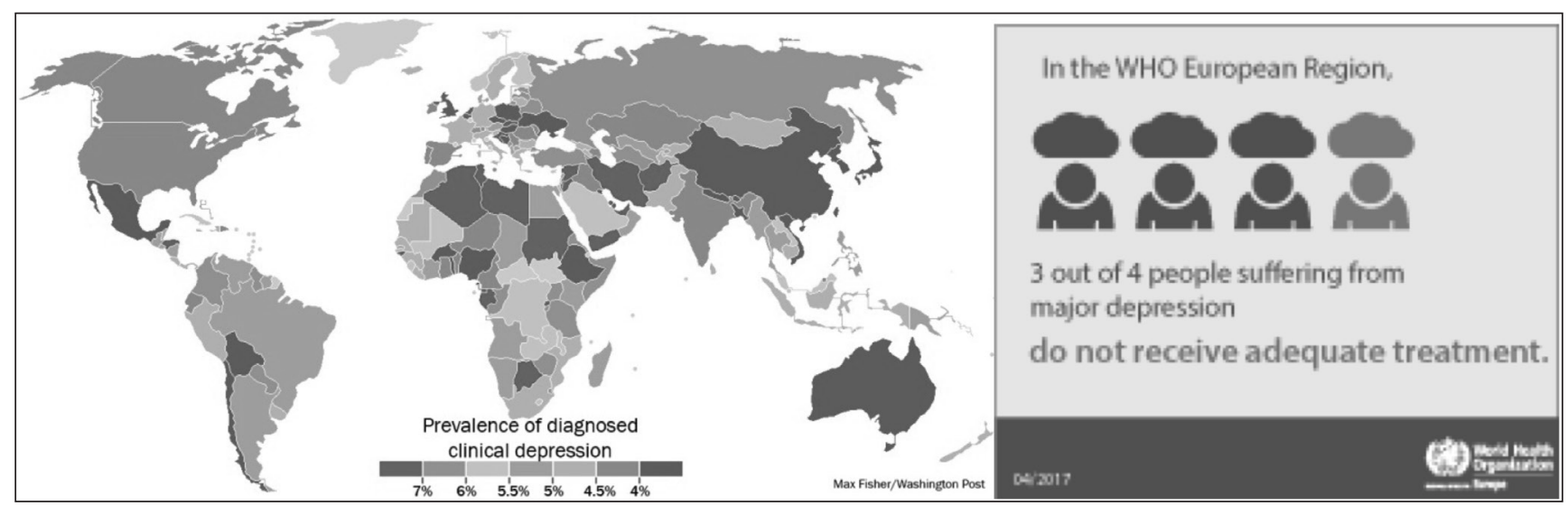

Abb. 7: Prävalenz in der Diagnose von psychischen Störungen versus fehlende Behandlungen.

In Europa machen psychische Gesundheitsprobleme fast 20 Prozent der Gesamterkrankungen aus und betreffen sogar jeden vierten Menschen im Laufe seines Lebens. Von den zehn Ländern mit den höchsten Selbstmordraten der Welt befinden sich neun in Europa. Trotz dieser Zahlen entsprechen die angebotenen Gesundheitsdienste häufig nicht den Anforderungen und Bedürfnissen der Patienten: Die Kluft zwischen der Pflege, die die Patienten benötigen würden, und dem, was sie erhalten, ist immer noch sehr gross. So wird beispielsweise die Hälfte der an Depressionen Erkrankten nie behandelt, und ganz allgemein werden sogar in Ländern mit einem entwickelten Gesundheitssystem 44-70 Prozent der an psychischen Pathologien Leidenden überhaupt nicht behandelt (Abbildung 7).

In einem Viertel der europäischen Länder sind Pflegedienste nicht einmal verfügbar, und in einigen Ländern sind mehr als 50 Prozent der Patienten in zu grossen und unzureichenden Strukturen untergebracht.

Wie aus dem Bericht der WHO 2013-2020 über die Prävention in Fragen der psychischen Gesundheit (http:// www.euro.who.int/en/de/health-topics/noncommunicable-diseases/mental-health/policy) hervorgeht, erfahren Menschen mit psychischen Störungen signifikant höhere Behinderungs- und Sterblichkeitsraten als der Durchschnitt.

Zum Beispiel haben Menschen mit schweren Depres- gilt ausserdem als erwiesen, dass psychische Störungen häufig mit Störungen durch Gebrauch von psychotropen Substanzen einhergehen.

Insgesamt bringen psychische, neurologische Störungen und psychotrope Substanzkonsumstörungen hohe Kosten mit sich, die 2004 weltweit 13 Prozent der globalen Krankheitslast (Global Burden of Disease) für die Gemeinschaft ausmachen. Depression allein macht 4,3 Prozent der globalen Krankheitslast aus und ist eine der Hauptursachen für Behinderungen weltweit (11\% der Lebensjahre, die weltweit mit einer Behinderung gelebt werden), insbesondere bei Frauen.

Die wirtschaftlichen Folgen dieser gesundheitlichen Beeinträchtigungen sind ebenso wichtig: Eine kürzliche Studie schätzt, dass sich die kumulativen Auswirkungen von psychischen Störungen in Bezug auf den Verlust von Wirtschaftsleistung zwischen 2011 und 2030 weltweit auf 16.300 Milliarden US-Dollar belaufen werden.

Die erste epidemiologische Studie über die Prävalenz psychischer Störungen ist Teil des europäischen Projekts European Study on the Epidemiology of Mental Disorders (ESEMeD), an dem sechs Länder (Italien, Belgien, Frankreich, Deutschland, Niederlande, Spanien) teilgenommen haben.

Die Studie gibt einen Überblick über die Häufigkeit von psychischen Störungen in der italienischen Bevölke- 
rung, die Beziehungen untereinander, die Assoziation mit möglichen Risikofaktoren und die Behandlungen, die in Anspruch genommen wurden (https://www.ncbi.nlm.nih. gov/pubmed/15128382).

Diese und andere Projekte wurden im Rahmen der WHO World Mental Health (WMH) Survey Initiative durchgeführt, an der sich mehr als 30 verschiedene Länder beteiligen. Das umfangreiche epidemiologische Forschungsprojekt wurde gemeinsam von WHO und der Universität Harvard (http://www.hcp.med.harvard. edu/wmh/) gefördert. Auf europäischer Ebene wurde das Projekt von der Europäischen Union und GlaxoSmithKline finanziert.

In der Schweiz leiden 27 Prozent der erwachsenen Bevölkerung (18-65) im letzten Jahr mindestens an einer von mehreren psychischen Störungen (u.a. Depressionen, Angstzustände, Ernährungsstörungen, Substanzabhängigkeit). Bei fast allen Erkrankungen - mit Ausnahme der Substanzabhängigkeit (5,6\% bei Männern, 1,3\% bei Frauen) - sind die Raten bei Frauen geringfügig höher als bei Männern. Die Prävalenzwerte dieser Krankheiten liegen in der Schweiz sogar über dem europäischen Durchschnitt. Sie kombinieren sich jedoch mit einem sehr effizienten, auf die Zugänglichkeit der Gesundheitsdienste ausgerichteten, föderalen Gesundheitssystem. Die Schweiz steht in Europa an erster Stelle hinsichtlich der Zugänglichkeit von Dienstleistungen und der Gesundheitsergebnisse nach dem Euro Health Consumer Index 2015, die jedes Jahr die europäischen Gesundheitssysteme klassifiziert.

Aus diesem Grund weist die Schweiz mit 30 Psychiaterinnen und Psychiatern pro 100.000 Einwohner eine der höchsten Zahlen in Europa auf, während der europäische Durchschnitt bei 9 liegt (vgl. Euro Health Consumer Index. http://www.healthpowerhouse.com/ index.php? Itemid=55).

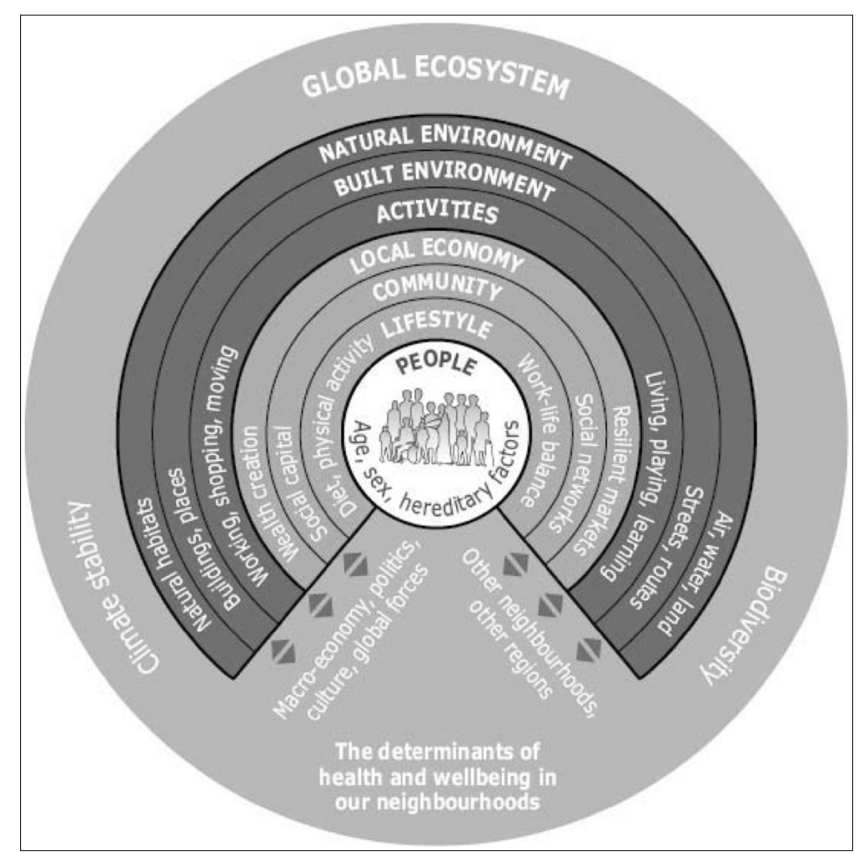

Abb. 8: Determinants of health and wellbeing. Quelle: Barton \& Grant.
Die Einstufung der psychischen Gesundheit als ein dringendes und integrales Problem weltweit ist von vorrangiger Bedeutung. Es sollte von den Institutionen mit der gleichen Dringlichkeit behandelt werden wie jede andere körperliche Störung, da die Ursachen klar identifiziert wurden, wie aus Abbildung 8 hervorgeht.

Psychische Störungen zeigen auch eine Komorbidität mit anderen Krankheiten, da sie zu gesundheitsschädlichen Verhaltensweisen wie Drogenmissbrauch, Drogen- bzw. Alkoholmissbrauch und Bewegungsmangel führen können. Depression ist heute bekannt als einer der wichtigsten Risikofaktoren für Bluthochdruck, HerzKreislauf-Erkrankungen und auch für Diabetes, der durch Essstörungen ausgelöst wird. Ganz zu schweigen von den gesundheitspsychologischen Problemen, das heißt dem Nachweis der Nicht-Reaktion auf Behandlungen (onkologisch, kardial), die hauptsächlich auf postoperative Depressionen zurückzuführen sind. Die nach wie vor geringen Kenntnisse in Bezug auf psychische Erkrankungen und die vorherrschenden kulturellen Vorurteile sind dafür verantwortlich, dass die meisten diagnostizierten Menschen nicht auf Behandlungen zurückgreifen, obwohl es für viele dieser Krankheiten wirksame Behandlungen gibt.

Diese sehr spezifischen soziokulturellen Aspekte psychischer Störungen erzeugen verheerende Auswirkungen aufgrund fehlender Diagnose: Krankheiten, die weniger schwerwiegend wären, wenn sie rechtzeitig erkannt und wirksam behandelt würden, werden deshalb chronisch. Eine der wichtigsten Herausforderungen an die öffentliche Gesundheit besteht deshalb darin, Risikofaktoren $\mathrm{zu}$ identifizieren, das Bewusstsein und die Kenntnis über die Wirksamkeit von Therapien zu stärken, die Stigmatisierung zu beseitigen, die in vielen Ländern noch immer mit psychologischer Behandlung verbunden ist und die Verfügbarkeit von tragbarem medizinischem Personal aus dem Gesundheitssystem für die primäre und sekundäre Prävention oder Früherkennung zu erhöhen, vor allem in den Bevölkerungsgruppen, die überproportional betroffen sind.

Die Einrichtungen des öffentlichen Gesundheitswesens müssen Präventionsprogramme für psychische Störungen in die allgemeine epidemiologische Überwachung (Surveillance) chronischer Krankheiten einbeziehen, um einige der in den WHO-Berichten (WHO) ermittelten Schlüsselaspekte angehen zu können.

1. Identifizierung von Personen mit psychischen Störungen $(95 \%$ aller Länder)

2. Früherkennung von Problemen (86\%);

3. Die Kapazität, Patienten mit Erkrankungen, die das Nervensystem und die psychische Behinderung betreffen, regelmässig zu behandeln $(85 \%)$.

4. Die Bereitstellung umfassender, integrierter und reaktionsfähiger Dienste für psychische Gesundheit und soziale Unterstützung auf territorialer Ebene.

5. Die Stärkung der Informationssysteme, der wissenschaftlichen Erkenntnisse und der Forschung im Bereich der psychischen Gesundheit

Vor dem Hintergrund dieser Überlegungen unterstreicht der Globale Aktionsplan 2013-2010 die Bedeutung der 
Annäherung an ein Gesundheitssystem, in dem die psychische Gesundheit geschätzt, gefördert und geschützt wird. Psychischen Störungen soll vorgebeugt werden und Menschen mit diesen Störungen müssen in der Lage sein, alle Menschenrechte in vollem Umfang wahrzunehmen und rechtzeitig Zugang zu qualitativ hochwertigen, kulturell angemessenen und qualitativ hochwertigen Gesundheitsund Sozialdiensten zu erhalten, die die Genesung fördern. Mit dem Ziel, ein möglichst hohes Gesundheitsniveau zu erreichen und uneingeschränkt, frei von Stigmatisierung und Diskriminierung, am sozialen und beruflichen Leben teilnehmen zu können.

\section{Präzision, Prävention: von Big Data bis zur individuellen Gesundheit}

In der modernen Gesellschaft hat die Evolution der biomedizinischen Datenproduktionstechnologie den Fokus auf «grosse Informationen» aus den «BIG DATA» verlagert. Die Biostatistik ist heute Teil der grösseren Disziplin der «Data Science». Technologische und biomedizinische Fortschritte haben einen rasch und kontinuierlich wachsenden Strom biologischer und Volksgesundheits-Daten sowie eine solide Wissensbasis geschaffen; diese hat das Potenzial hat, die Gesundheitsversorgung durch wirksamere und individuellere Ansätze zur Prävention, Diagnose und Behandlung von Krankheiten zu verbessern. Ausserdem trägt die Fähigkeit dazu bei, die Erbringung von Gesundheitsdienstleistungen effizienter zu gestalten und Innovation und Wirtschaftswachstum zu unterstützen.

Die BIG DATA beziehen sich auf die Menge an Informationen, ihre Komplexität und die Geschwindigkeit, mit der sie genutzt werden können. Tatsächlich werden die "grossen» biomedizinischen Daten nach den sogenannten 3V klassifiziert: "Volume», das sich auf die Anzahl der Stichproben und die Anzahl der Variablen und Merk-

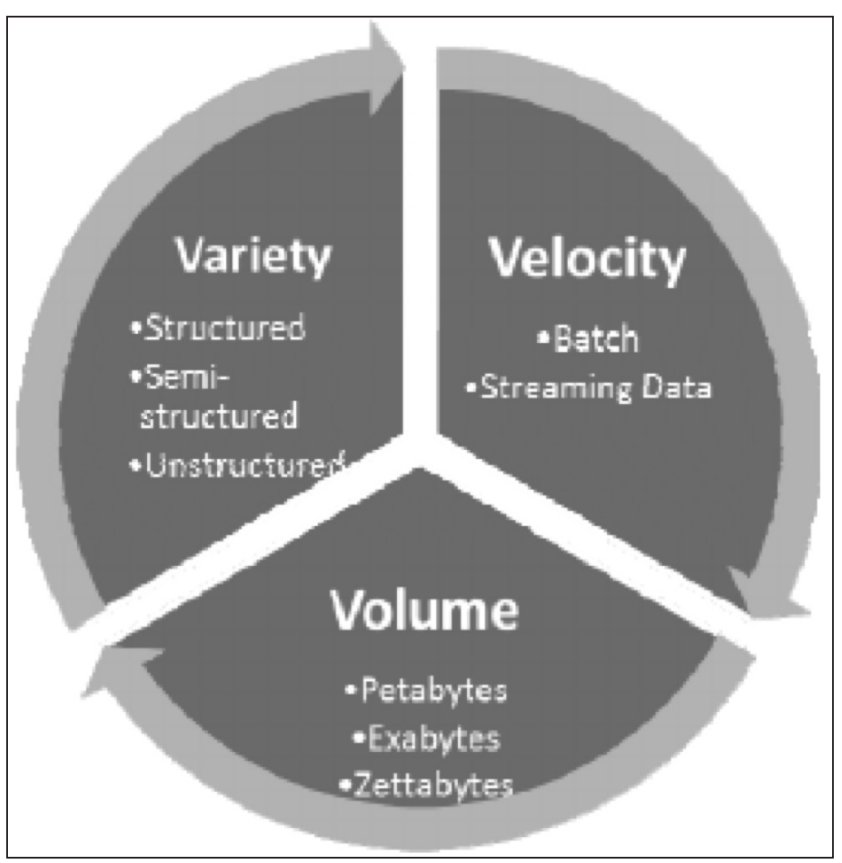

Abb. 9: Die Architektur des 3 V-Modells für Big Data. male pro Stichprobe bezieht. Dieser Aspekt betrifft zum Beispiel die Bildung grosser internationaler Konsortien zur Sammlung biomedizinischer Daten, an denen verschiedene Gesundheitsorganisationen, Krankenhäuser und Forschungszentren beteiligt sind. "Velocity» bezieht sich auf die Geschwindigkeit der Datengenerierung im Zusammenhang mit neuen Technologien und «Variety» bezieht sich auf verschiedene Datenquellen wie klinische, genomische und biomolekulare Daten, wie in Abbildung 9 dargestellt wird.

Der in Abbildung 10a und b dargestellte sogenannte «Garnter Hyper Cycle» (Hype-Zyklus) repräsentiert die Lebens- und Entwicklungsphasen wissenschaftlicher Entdeckungen über die Zeit sehr gut. Insbesondere ist er bezeichnend für die Tatsache, dass wir für die «Big Data» heute fast den Gipfel der sogenannten «technologischen Erwartungen» erreicht haben.

Man ahnt sofort, wie einer der «heikelsten» Aspekte des Beitrags der BIG DATA zum biomedizinischen Wissen das betrifft, was viele als das vierte «V» oder als «Veracity» (Wahrhaftigkeit) bezeichnen: Wie viel «gute Information» lässt sich aus den "BIG DATA» ableiten?

Inwieweit können wir diese Daten, die ohne experimentelle Planung erhoben wurden und über deren Qualität wenig Informationen vorliegen, dazu verwenden, in «kausaler» Hinsicht Rückschlüsse auf Pathologien oder Risikofaktoren zu ziehen, zumal sie ja bereits in allen Beobachtungsstudien komplexe Aspekte darstellen, die es zu behandeln gilt?

Die Nutzung von BIG Data in den biomedizinischen Wissenschaften ist auch unter dem Gesichtspunkt der öffentlichen Gesundheit und Bioethik sehr aktuell geworden. 2015 widmete der Nuffield Council on Bioethics dem Thema "The collection, linking and use of data in biomedical research and health care: ethical issues» einen ganzen Abschnitt. In diesem Zusammenhang wurde das wachsende öffentliche Interesse an einem verantwortungsvollen Umgang mit Daten deutlich, die Wissen generieren, Innovationen fördern und zur Verbesserung der Gesundheit. Dazu gesellen sich berechtigte Bedenken hinsichtlich des Schutzes der Privatsphäre.

Im Zusammenhang mit Transparenz, Datenschutz und Einverständniserklärung taucht eine Reihe kritischer

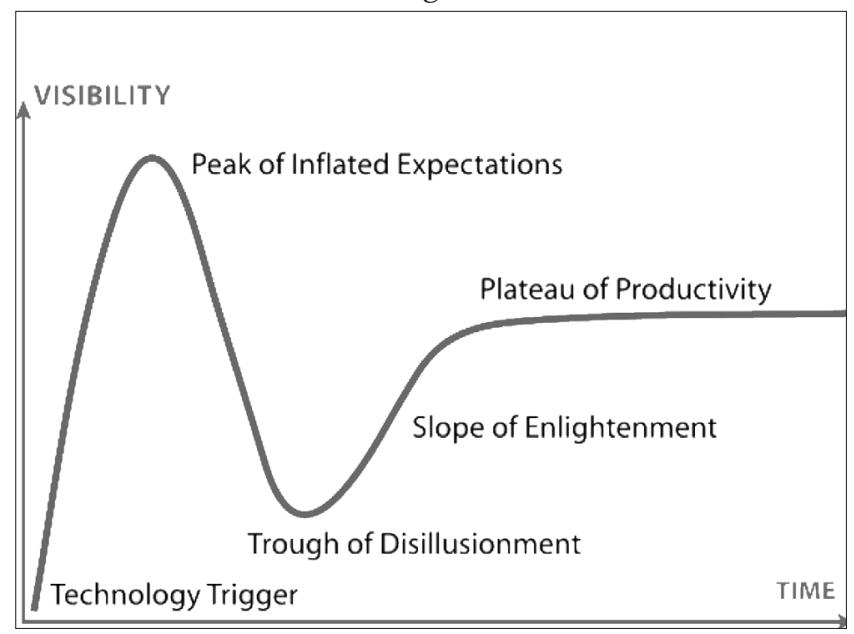

Abb. $10 a$ 


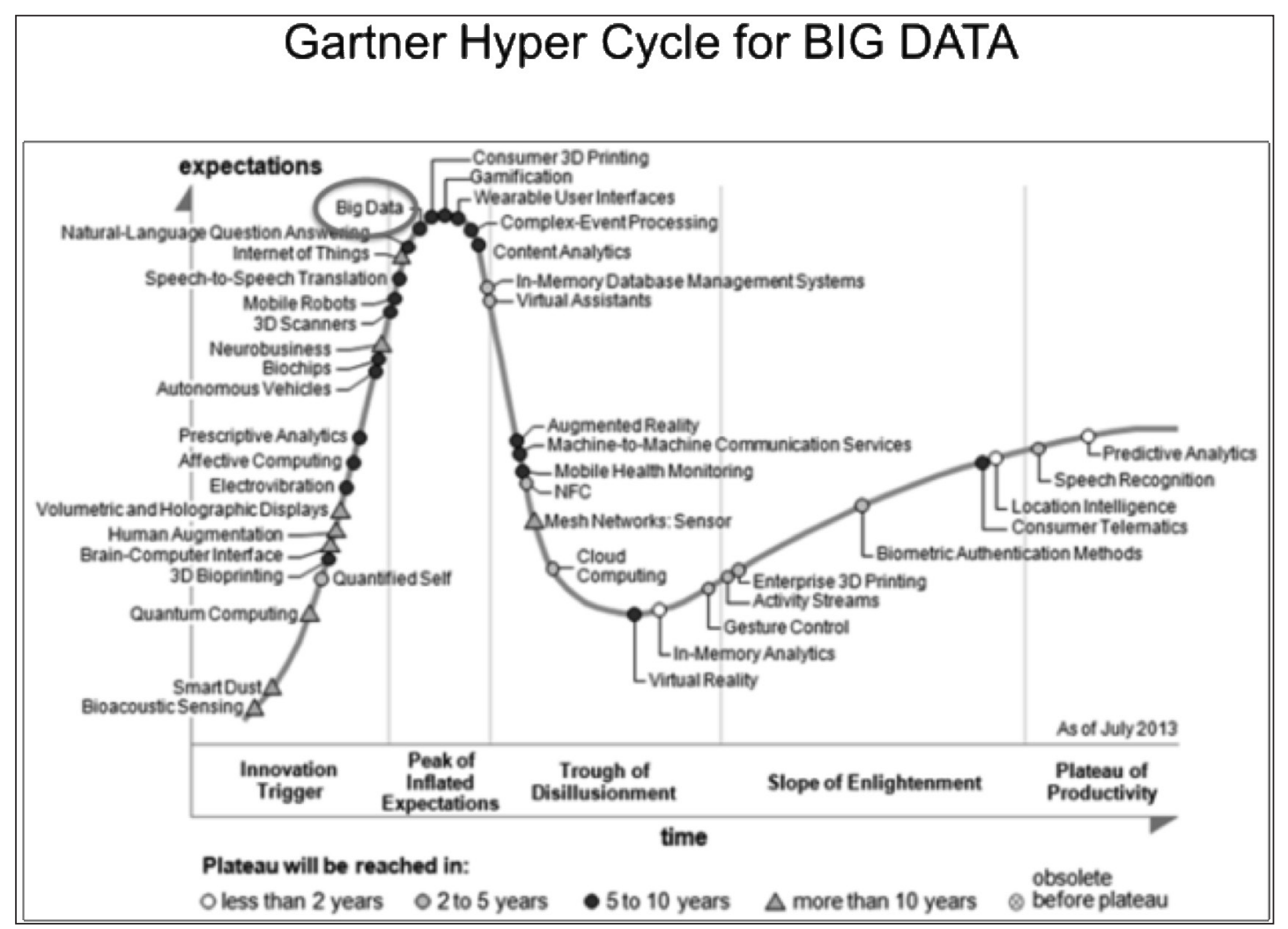

Abb. 10b:

Gartner Hype

Cycles bieten

eine grafische

Darstellung des

Reifegrades und

der Akzeptanz

von Technolo-

gien und Appli-

kationen sowie

deren poten-

zielle Relevanz.

Fragen auf, die angemessen angegangen werden sollte. Es geht darum, sicherzustellen, dass diese enorme Datenverfügbarkeit und alle daraus resultierenden Veränderungen des globalen Wissens besser verwaltet und genutzt werden. Ab 2012 werden täglich ca. 2,5 Exabyte Daten erzeugt (Zahl, die sich in den nächsten Jahren voraussichtlich verdoppeln wird) und dass eine unsachgemässe Verwendung dieser riesigen Datenmenge Zusammenhänge mit Krankheiten offenlegen könnte, so dass eine Person für bestimmte Berufe oder bestimmte Voraussetzungen für Kranken- oder Lebensversicherung als ungeeignet eingestuft wird.

In der Tat entsteht neu die sogenannte «Digital Epidemiology», die aus einer neuen Generation von Systemen zur Überwachung der öffentlichen Gesundheit besteht. Diese agieren über internationale Grenzen hinweg und integrieren traditionelle Systeme, indem sie den weit verbreiteten Zugang zum Internet und das explosionsartige Wachstum mobiler Geräte und Online-Plattformen nutzen.

Mit Hilfe globaler Daten in Echtzeit verspricht die digitale Epidemiologie beispielsweise, den Infektionsherd einer Krankheit frühzeitig zu erkennen. Der jüngste Fall ist die Ebola-Epidemie in Westafrika im Jahr 2014 zeigt: Es geschieht heute viel schneller als früher, zum Beispiel als John Snow im Jahr 1854 die Cholera nach monatelanger Arbeit entdeckt hat.

Das Sammeln, Verknüpfen und Nutzen von Daten in der biomedizinischen Forschung und im Gesundheitswesen: ethische Fragen (http://nuffieldbioethics.org/project/ biological-health-data/)

\section{Schlussfolgerungen}

Die «Daten»-Technologie ist parallel zu den biomedizinischen Technologien vorangeschritten und hat das Konzept der epidemiologischen Analyse, der prädiktiven Analyse und der Überwachung in integrierte Teile eines einzigen Prozesses verwandelt, der der Prävention und den Massnahmen im Bereich der öffentlichen Gesundheit zugrunde liegt.

So wie die Präzisionsmedizin die Daten einzelner Patienten nutzt, um die am besten geeignete therapeutische Massnahme zu planen, so nutzt die präzise Prävention «Big Data», um die Auswirkungen von Risikofaktoren auf die Entwicklung bestimmter Krankheiten innerhalb einer Bevölkerung zu untersuchen und Strategien zu identifizieren, um deren Ausbruch zu verhindern oder die Nutzung bestehender therapeutischer Praktiken und Ressourcen zu optimieren.

Auf politischer Ebene ist daher erforderlich, auf Präventionsstrategien zu fokussieren, die Ärzte, Epidemiologen, Genetiker und Gesundheitsorganisationen wirkungsvoll umsetzen könnten. Durch Datenzugang wären sie in der Lage, diese enorme Datenmenge mit hoher Geschwindigkeit, Komplexität und Variabilität zu durchkreuzen und interagieren zu lassen. Ausserdem könnte diese grosse Menge an Daten über Einzelpersonen zu ethischen und rechtlichen Problemen führen, die bei der Planung von Bevölkerungsstudien berücksichtigt werden müssen.

Es trifft durchaus zu, dass aus der Integration verschiedener Datenquellen grundlegende Inputs für die Identifizierung von Risikofaktoren, neuen Biomarkern oder Infektionsherden im Zusammenhang mit neuen Viren entstehen können. Dennoch bleiben die Bewertung 
der Einstellung und des Verhaltens der Leute gegenüber der Gesundheit und die Pharmakovigilanz nach wie vor von primärem Interesse.

\section{Literatur}

de Moivre, A. (1725). Annuities upon Lives: or, The Valuation of Annuities uon any Number of Lives; as also, of Reversions. To which is added, An Appendix concerning the Expectations of Life, and Probabilites of Survivorship. London: Fayram, Motte and Pearson.

Eurostat (2006). EU integration seen through statistics. Luxembourg: Publications of the European Union. http://epp.eurostat.ec.europa. eu/cache/ITY_OFFPUB/KS-71-05-691/EN/KS-71-05-691-EN.PDF (18.03.2013).

Gompertz, B. (1825). On the nature of the function expressive of the law of human mortality. Philosophical Transactions of the Royal Society of London, Series A 115, 513-580.

Hald, A. (1990). A History of Probability and Statistics and Their Applications before 1750. New York: Wiley.

Makeham, W.M. (1860). On the law of mortality, and the construction of mortality tables. Journal of the Institute of Actuaries, 8, 301

Makeham, W.M. (1860). On the law of mortality, and the construc tion of mortality.

Neff, G. (2013). Why big data won't cure us. Big Data, 1, 117-123. pmid:25161827.

Salathé, M., Bengtsson, L., Bodnar, T.J., Brewer, D.D., Brownstein, J.S. et al. (2012). Digital epidemiology. PLoS Comput Biol, 8: e1002616. pmid:22844241.

Stjernsward, J. (WHO) (1989). Battle against tobacco. Editorial. Journal of the National Cancer Institute, 81,1524-1525.

United Nations Economic Commission for Europe (2010). Regional Ministerial Conference on Ageing will review 10 years of implementation of Madrid International Plan of Action on Ageing. Genf: UNECE. http://www.unece.org/press/pr2010/10env_p40e.html (19.07.2013).

Vayena, E., Salathé, M., Madoff, L.C. \& Brownstein J.S. (2015). Ethical Challenges of Big Data in Public Health. PLoS Comput Biol, 11(2): e1003904.

\section{New challenges of epidemiology in political and social contexts. From public health service to prevention policies}

This contribution is addressed to provide a large spectrum overview on how the evolution of biomedical information has brought unavoidably about a change of tools and modes of analysis both from the epidemiology and public health service point of view. In order to face the challenges connected to the change of population dynamics, to the large migration fluxes, the change of ageing dynamics and the very rapid evolution of medicine towards "precision medicine», it is necessary that the social-organizational response of social and health system adapts itself to the ongoing changes and new needs. In this article we will show how, moving from an individual perspective to the one of a population typical of epidemiology, a lot of paradigms connected to data also change and this positions us in a "global system» model type where the integration of different sources of information and prevention interventions become unavoidable.

Keywords: Public Health, surveillance, Ageing, big data, mental health

\section{Die Autorin}

Prof. Clelia Di Serio, Professorin für medizinische Statistik und Epidemiologie. 1991: Hochschulabschluss in Volkswirtschaft mit Fachrichtung Statistik. 1995: Executive Master in Statistics (UNC-USA) 1992-1996. Promotion in Statistik (Chapel Hill, PhD-Programm University of North Carolina-USA gemeinsam mit der University of Trento). Seit 2007 Professorin für Statistik an der Universität der italienischen Schweiz in Lugano. Seit 2005 Direktorin des CUSSB (Universitätszentrum für Statistik in den biomedizinischen Wissenschaften). Veröffentlichungen einsehbar unter: http://www.unisr.it/k-teacher/diserio-mariacleliastefania/ \#1456841975415-dfc853c9-c0d3.

\section{Kontakt}

diserio.clelia@hsr.it clelia.di.serio@usi.ch

Centro Universitario di Statistica per le Scienze Biomediche (CUSSB: www.cussb.unisr.it)

Università Vita-Salute San Raffaele

Via Olgettina 58

20132 Milano

Università della Svizzera Italiana, Via Buffi, Lugano. 